\title{
HPV type-specific risks of high-grade CIN during 4 years of follow-up: A population-based prospective study
}

\author{
P Naucler', W Ryd ${ }^{2}$, S Törnberg ${ }^{3}$, A Strand ${ }^{4}$, G Wadell ${ }^{5}$, BG Hansson', E Rylander ${ }^{6}$ and J Dillner,, \\ 'Department of Medical Microbiology, MAS University Hospital, Lund University, S-20502 Malmö, Sweden; ${ }^{2}$ Department of Pathology and Clinical \\ Cytology, Sahlgrenska University Hospital, 41345 Göteborg, Sweden; ${ }^{3}$ Cancer Screening Unit, Oncologic Centre, Karolinska Hospital, 17176 Stockholm, \\ Sweden; ${ }^{4}$ Department of Medical Sciences, Dermatology and Venereology, University Hospital, 75185 Uppsala, Sweden; ${ }^{5}$ Department of Virology, \\ University of Northern Sweden, 90187 Umeå, Sweden; ${ }^{6}$ Institute of Clinical Sciences, Department of Obstetrics and Gynecology, Karolinska Institute, \\ Danderyd Hospital, 18288 Stockholm, Sweden
}

\begin{abstract}
We followed a population-based cohort of 5696 women, 32-38 years of age, by registry linkage with cytology and pathology registries during a mean follow-up time of 4.1 years to assess the importance for CIN2 + development of type-specific HPV DNA positivity at baseline. HPV 16, 31 and 33 conveyed the highest risks and were responsible for $33.1,18.3$ and $7.7 \%$ of CIN2 + cases, respectively. Women infected with HPV I8, 35, 39, 45, 5I, 52, 56, 58, 59 and 66 had significantly lower risks of CIN2 + than women infected with HPV 16. After adjustment for infection with other HPV types, HPV types 35, 45, 59 and 66 had no detectable association with CIN2 +. In summary, the different HPV types found in cervical cancer show distinctly different CIN2 + risks, with high risks being restricted to HPV 16 and its close relatives HPV 31 and HPV 33.
\end{abstract}

British Journal of Cancer (2007) 97, 129-132. doi:I0.1038/sj.bjc.6603843 www.bjcancer.com

Published online 5 June 2007

(c) 2007 Cancer Research UK

Keywords: cervical cancer; screening; papillomavirus; attributable proportion; cohort studies

Infection with 'high-risk' types of HPV is the major cause of cervical cancer, and the distribution of different HPV types in cancer tissue has been extensively analysed (Walboomers et al, 1999; Bosch et al, 2002; Munoz et al, 2003). Fifteen HPV types that infect the genital mucosa have been proposed as 'high-risk' HPV types as they have been found more often in cervical cancers than among healthy subjects (Munoz et al, 2003). Data on the HPV type-specific risk of cervical neoplasia has hitherto mostly been based on cross-sectional case-control studies (Bosch et al, 1995; Clifford et al, 2003; Munoz et al, 2003). Case-control studies are sensitive to several biases, notably selection bias, differential sampling bias and other reverse causality biases. Absolute risks and population attributable proportions for each HPV type form the basis for decisions regarding which HPV types should be included in HPV screening tests as well as in vaccines and it is therefore important to estimate the type-specific risks using population-based prospective studies, a study design that minimises major sources of bias.

Only two previous prospective studies have assessed the risk associated with several individual HPV types (Schiffman et al, 2005; Berkhof et al, 2006), while several prospective studies have assessed certain clusters of HPV types (Koutsky et al, 1992; Liaw et al, 1999; Sherman et al, 2003; Szoke et al, 2003; Peto et al, 2004; Winer et al, 2005) or only investigated HPV types 16 and 18 (Khan et al, 2005).

\footnotetext{
*Correspondence: Professor J Dillner;

E-mail: joakim.dillner@med.lu.se

Revised II May 2007; accepted 16 May 2007; published online 5 June 2007
}

Therefore, we HPV-tested a population-based cohort of women and used a comprehensive registry-based follow-up to identify the risks for development of histopathologically verified high-grade cervical intraepithelial neoplasia (CIN2 + ) associated with infections with 14 different so-called 'high-risk' HPV types.

\section{MATERIALS AND METHODS}

\section{Cohort definition}

A population-based multicentre study was started in Sweden in May 1997 with the main purpose to evaluate the effect of HPV testing in primary cervical cancer screening. Women aged between 32 and 38 years (mean age: 35.1 years) in five regions in Sweden (Gothenburg, Malmö, Stockholm, Umeå and Uppsala) who took part in organised cervical screening were invited to take part in the study. Following informed consent, 12527 women were enrolled and randomised either to action on HPV tests (6257 women) or to no action on HPV tests (6270 women), as described in detail elsewhere (Elfgren et al, 2005). All women had a cervical brush sample taken at baseline that was used for routine cytological screening and then frozen in $1 \mathrm{ml}$ of $0.9 \% \mathrm{NaCl}$ for future $\mathrm{HPV}$ DNA analysis. Referral to colposcopy was based on routine clinical management. Furthermore, HPV-positive women in the intervention arm that did not have an abnormal enrolment smear in cytology and pathology registries were invited for a second HPV test and cytology on average 19 months later and if persistently positive invited to colposcopy. A matched number of women from the control arm were also invited for HPV test, cytology and 
colposcopy (Elfgren et al, 2005). For the present study, a population-based cohort was formed from 6257 women in the intervention arm as well as 409 women randomly selected from the control arm that had HPV tests performed on their baseline samples.

All women were followed by registry linkages using unique personal identification numbers with both the regional cytology and pathology registries in the enrolling regions, as well as with the national cervical screening registry, to detect development of $\mathrm{CIN} 2+$. All women with an abnormal histopathological diagnosis as well as all women invited for colposcopy within the study protocol had their specimens re-evaluated by a single expert pathologist (WR) who was masked to the HPV status of the women. For 22 specimens that could not be located in the pathology archives, the original diagnosis was retained. Overall, $126 / 148(85 \%)$ of the CIN2 + diagnoses in the present study have been confirmed by expert pathologist review. Women who had an inadequate ( $\beta$-globin-negative) HPV test at baseline (172 women) or who had no cytological or histological samples registered during follow-up (796 women) were excluded from the analysis. The final population-based cohort thus consisted of 5696 women with a mean follow-up time of 4.1 years.

\section{HPV DNA testing}

The virus laboratory that performed the HPV analyses was masked to cytological and histological diagnoses of the women. Cervical brush samples were analysed using a general HPV primer GP5 + I $6+-$ mediated PCR-enzyme immunoassay consisting of a pool of digoxigenin-labelled HPV type-specific oligonucleotide probes of 14 high-risk HPV types (types 16, 18, 31, 33, 35, 39, 45, 51, 52, 56, 58, 59, 66 and 68) (de Roda Husman et al, 1995; Jacobs et al, 1997). Human $\beta$-globin was amplified simultaneously in the PCR-EIA assay to test for sample DNA quality. All HPV-positive samples were typed by reverse dot blot hybridisation (RDBH) using HPV type-specific plasmids corresponding to the different HPV types tested for in the PCR-EIA (Forslund et al, 2002). PCR-EIA-positive samples negative in RDBH were cloned and sequenced. Samples were considered HPV positive only if successfully typed by RDBH or by DNA sequencing.

\section{Statistical analysis}

Statistical analyses were performed using STATA 9.0. Absolute cumulative risks of future $\mathrm{CIN} 2+$ and $\mathrm{CIN} 3+$ with binominal exact $95 \%$ confidence intervals (CI) were calculated for each HPV type. Relative rates for CIN2 + and their 95\% CI were calculated using Poisson regression. Women were censored at their last testing date, except for women who developed CIN2 + who were censored at the date when the diagnostic biopsy was taken. Since lesions that were detected early during follow-up might have been present already at baseline we split the data according to length of follow-up of more or less than 6 months. A likelihood ratio test was then performed to assess if there was interaction between HPV infection and time of CIN2 + diagnosis. There was weak evidence for interaction $(P$-value 0.10$)$ and thus we adjusted for time of diagnosis by introducing a term in the regression model representing follow-up of more or less than 6 months. Because the risk associated with one HPV type can be confounded by coinfection with other HPV types, we adjusted for infection with other HPV types by including type-specific HPV data as single variables in a multivariate regression model. Population attributable proportions were calculated as $\mathrm{p}_{c}{ }^{*}(\mathrm{RR}-1) / \mathrm{RR}$ where $\mathrm{p}_{\mathrm{c}}$ is the proportion exposed to an HPV type among cases and RR is the type-specific relative rate adjusted for infection with other HPV types and censoring before or after 6 months of follow-up (Miettinen, 1974). Ninety-five percent CI for population attributable proportions were calculated from the standard error of the log-transformed complement of the population attributable proportions $[\ln (1-\mathrm{PAF})]$ (Rothman and Greenland, 1998). To test for type-specific differences of relative rates, the $\beta$-coefficients of each HPV type in the multivariate regression model were compared with the $\beta$-coefficient of HPV 16 by fitting a constrained model and performing a likelihood ratio test (StataCorp, 2005).

\section{RESULTS}

During a mean follow-up time of 4.1 years, 148 women developed CIN2 + . One hundred and twenty-seven $(85.8 \%)$ of these had a positive HPV test at baseline. The HPV type with highest population-based prevalence of infection was HPV $16(2.5 \%)$, followed by type $31(1.4 \%), 45(0.9 \%)$ and $18(0.7 \%)$. A single HPV type was detected in $401(87.0 \%)$ of the HPV-positive women, while $60(13.0 \%)$ women had more than one virus type detected in the same sample. Most multiple HPV infections were infections with two HPV types, but up to four different HPV types could be detected in the same sample. The absolute risk of CIN2 + among HPV-positive women ranged from 0\% for HPV 59 to $48.0 \%$ (95\% CI: 27.8-68.7) for HPV 33 (Table 1). HPV 16 infection had an absolute risk of future CIN2 + of 36.6\% (95\% CI: $28.7-45.1$ ) and HPV 18 infection had an absolute risk of $25.6 \%$ (95\% CI: $13.0-$ 42.1). HPV 31 and 58 were also associated with very high absolute risks of $36.7 \%$ (95\% CI: $26.1-48.3$ ) and $30.4 \%$ (95\% CI: $13.2-52.9$ ), respectively (Table 1 ). The absolute risk of developing CIN2 + among women infected with any high-risk HPV type was $27.5 \%$ (95\% CI: 23.5-31.9). Women who were HPV negative had a low absolute risk of future CIN2 $+(0.40 \%$ (95\% CI: $0.25-0.61))$. The absolute risks for future CIN3 + were highest for HPV $16(28.2 \%$ (95\% CI: $20.9-36.3))$, HPV 33 (28.0\% (95\% CI: $12.1-49.4)$ ), HPV 31 (22.8\% (95\% CI: $14.1-33.6))$ and HPV 58 (21.7\% (95\% CI: 7.5-43.7)) (Table 1).

The relative rates of $\mathrm{CIN} 2+$ for each HPV type were assessed using women negative for the corresponding HPV type as the reference group (Table 1). It should be noted that we have deliberately avoided analyses using women who were HPV negative for all HPV types as the reference group. Such analysis cannot take the issue of multiple infections into account, resulting in inflated relative rates, for example the relative rate for HPV 16 was $27.6(19.7-38.6)$ (Table 1), but the HPV 16-related relative rate would have been $118.4(71.3-196.6)$ if women negative for all HPV types had been used as a reference group.

After adjustment for concomitant infections with other HPV types, the type-specific relative rates segregated into three groups. HPV 16, 31 and 33 had very high relative rates (exceeding 10) (Table 1), with all other types having significantly lower risks than HPV 16 (Table 1). HPV types 18, 39, 51, 52, 56 and 58 conferred significantly elevated risks for $\mathrm{CIN} 2+$, with relative risks in the range 3- to 7-fold (Table 1). We were not able to detect any excess risk for CIN2 + associated with HPV 35, 45, 59 or 66 (Table 1).

Population attributable proportions were based on the typespecific relative rates adjusted for all other HPV types. HPV 16 attributed to $33.1 \%$ (95\% CI: $24.7-40.6)$ followed by HPV 31 (18.3\% (95\% CI: 11.5-24.5)), HPV 33 (7.7\% (95\% CI: $3.1-12-0)$ ) and HPV 18 (5.7\% (95\% CI: 1.5-9-7)) (Table 1). HPV 35, 39, 45, 56,59 and 66 each contributed to $2.0 \%$ or less of CIN2 + in the population. HPV 16 and 18 together attributed to $39.0 \%$ (95\% CI: $30.2-46.7$ ) of CIN2 + and the four types (HPV 16, 18, 31, 33) that contributed most individually jointly attributed to $64.0 \%$ (95\% CI: $55.0-71.2$ ). Overall, $84.7 \%$ (95\% CI: 73.3-89.7) of CIN2 + was attributed to an HPV infection.

\section{DISCUSSION}

This prospective large-scale study provides individual estimates of the risk for future histopathologically confirmed high-grade CIN 
Table I HPV type-specific risks and population attributable proportions for the development of histopathologically confirmed high-grade CIN during a mean follow-up time of 4 years in a cohort of 5696 women

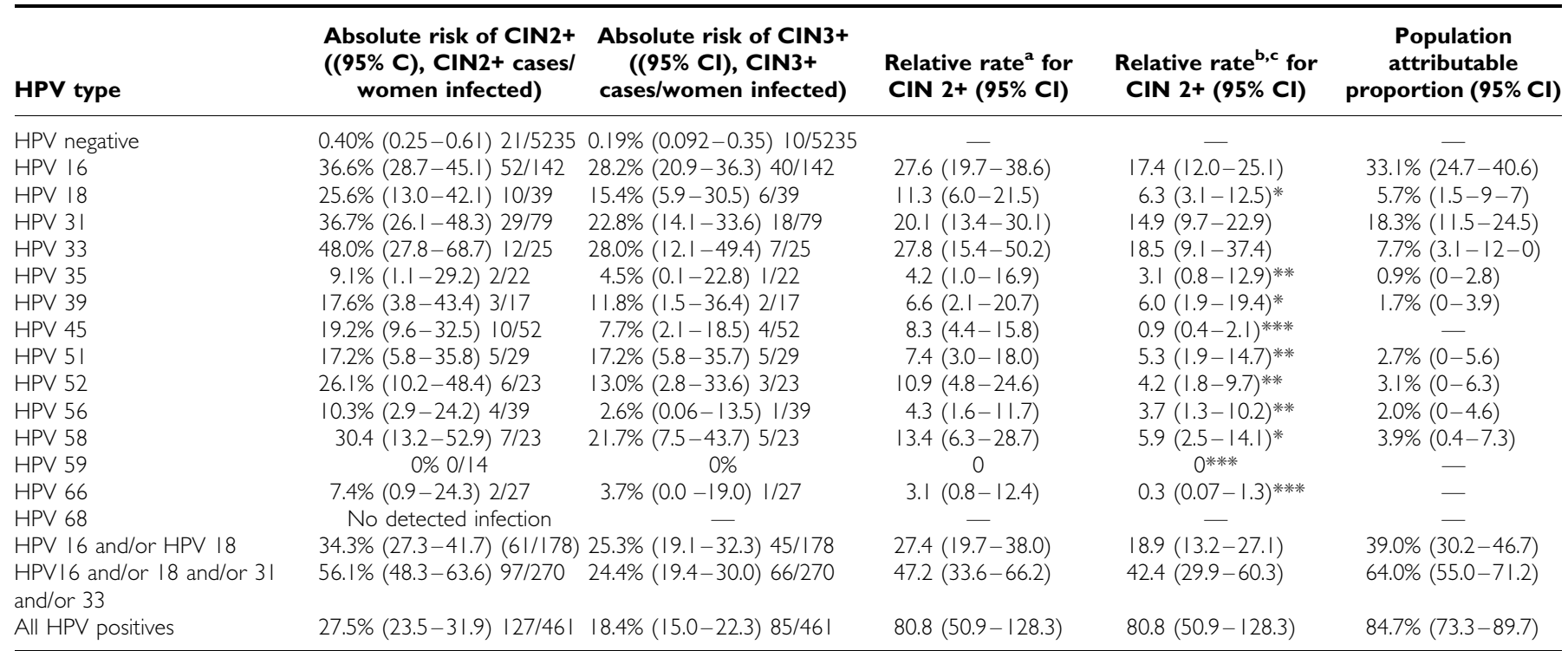

${ }^{a}$ Adjusted for censoring before or after 6 months of follow-up. ${ }^{b}$ Adjusted for censoring before or after 6 months of follow-up and for other HPV types. ${ }^{\mathrm{C}}$ Type-specific relative rates that were significantly different from HPV I6 relative rate: $P$-value $* 0.01<0.05, * * 0.001<0.01$ and $* * * * 0.001$.

for each one of 14 genital HPV types, commonly included in 'highrisk' HPV tests. The large size and prospective study design, nested within the national screening programme and our comprehensive follow-up argue in favour of reliability of estimates.

We report that several HPV types previously classified as 'highrisk' types (Munoz et al, 2003) convey significantly lower risk for future CIN2 + compared to HPV 16. This is in line with a cohort study in Costa Rica of 10000 women which reported HPV 16 to be uniquely carcinogenic among 'high-risk' HPV types in its risk of progression to CIN3/cancer after persistent infection (Schiffman et al, 2005). Also, in a cohort of 20810 women in Portland, US, the 10 -year cumulative incidence rate of CIN3 + among women with a negative, equivocal, or mildly abnormal baseline cervical pap smear was $17.2 \%$ among HPV 16-positive women, $13.6 \%$ among HPV 18-positive women but only 3.0\% among women with a positive Hybrid Capture 2 test but negative for HPV 16 and 18 at baseline (Khan et al, 2005). Besides HPV 16 we also found that HPV 31 and 33 conveyed rate ratios of future CIN2 + above 10. These results are similar to a Dutch report, where these three types were associated with the highest risk for future CIN2 + during 18 months of follow-up (Berkhof et al, 2006).

Risk classification of genital HPV types has hitherto mostly been based on case-control studies (Munoz et al, 2003). In a pooled analysis of 11 case-control studies performed by IARC that included samples from 1918 women with squamous cervical carcinoma and 1928 controls, 15 HPV types (HPV 16, 18, 31, 33, $35,39,45,51,52,56,58,59,68,73$ and 82 ) were classified as highrisk types and HPV 26, 53 and 66 as probably high-risk types (Munoz et al, 2003). Even though prospective studies use highgrade CIN as end point, which is only a surrogate for cervical cancer, they convey important methodological advantages to establish causal relationships. Case-control studies are not only sensitive to reverse causality bias, but many case-control studies sample differently from cases (biopsies) and controls (brush samples) which might affect HPV test performance. Furthermore, it is often difficult to obtain population-representative controls. Therefore we believe that more robust risk classification of HPV types can be obtained from prospective population-based studies.

Population-attributable proportions estimate the reduction in incidence of a disease if the exposure under study was eliminated from the population (e.g., by vaccination). The populationattributable proportions were based on HPV type-specific relative rates, that is the rates for a specific HPV type compared to the rate among women negative for that HPV type (not compared to women negative for all HPV types). This choice of reference group is more appropriate for studying attributable proportions since the elimination of one HPV type does not imply that the population would become negative for all HPV types. It should be noted that even though our type-specific relative rates as well as our classification of which types are oncogenic differs from the above-mentioned studies (Munoz et al, 2003), our relative rates, if we would have used women negative for any HPV as reference group would have been rather similar to these other studies (data not shown).

After adjustment of our type-specific estimates for presence of other HPV types, we found no elevated risk for future CIN2+ among women infected with HPV 35, 45, 59 and 66. However, the CI were large, especially for HPV 35 , and it is possible that our failure to detect any association with disease for these HPV types may be attributable to limited statistical power. We found that the elimination of HPV 16 would diminish the burden of CIN2+ by $33 \%$ and removal of both HPV 16 and 18 would reduce the incidence of CIN $2+$ by $39 \%$ in our population of Swedish women aged $32-38$ years. It is noteworthy that both HPV 31 and 33, which are not included in current prophylactic vaccines, both contributed to more CIN2 + than did HPV 18. These four HPV types (HPV 16, 18, 31 and 33) jointly attributed to almost $65 \%$ of all CIN2 +, and prevention of infection with these four types would therefore be most important. By contrast, HPV types 35, 39, 45, 56, 59 and 66 each contributed to $2.0 \%$ or less of CIN $2+$ in the population and inclusion of these types in HPV vaccines therefore seems less important, as the theoretical possibility exists that inclusion of too many HPV types in second generation HPV vaccines might impair the response against the most important HPV type (i.e., HPV 16). In a cohort of 10000 women in Costa Rica, HPV 16 was the type that contributed most to prevalent cases of CIN3 + followed by HPV 58, 18 and 31 (Schiffman et al, 2005). In our study, HPV 58 infection was rare in the population and only $4 \%$ of CIN $2+$ cases were attributed to HPV 58 . Since population attributable proportions are dependent not only on the 
type-specific relative risks but also on the type-specific prevalence, it is likely that differences in type-specific prevalences of infection in different populations account for the observed differences.

Limitations of the study include the risk of verification bias, as women with a negative HPV test are less likely to be referred to colposcopy and subsequently having a biopsy taken during the course of the study. This could influence the attributable proportion estimates but not the relative comparison of typespecific absolute risks or risk rates. The bias of HPV-based verification diminishes successively with increasing length of follow-up using conventional cytology and most of the women in the cohort were followed for more than two screening rounds (in Sweden the screening interval is 3 years). Also, detection of $\mathrm{CIN} 2+$ is exceedingly rare when HPV-negative, cytology-negative

\section{REFERENCES}

Belinson J, Qiao YL, Pretorius R, Zhang WH, Elson P, Li L, Pan QJ, Fischer C, Lorincz A, Zahniser D (2001) Shanxi Province Cervical Cancer Screening Study: a cross-sectional comparative trial of multiple techniques to detect cervical neoplasia. Gynecol Oncol 83: 439-444

Berkhof J, Bulkmans NW, Bleeker MC, Bulk S, Snijders PJ, Voorhorst FJ, Meijer CJ (2006) Human papillomavirus type-specific 18-month risk of high-grade cervical intraepithelial neoplasia in women with a normal or borderline/mildly dyskaryotic smear. Cancer Epidemiol Biomarkers Prev 15: $1268-1273$

Bosch FX, Lorincz A, Munoz N, Meijer CJ, Shah KV (2002) The causal relation between human papillomavirus and cervical cancer. J Clin Pathol 55: 244-265

Bosch FX, Manos MM, Munoz N, Sherman M, Jansen AM, Peto J, Schiffman MH, Moreno V, Kurman R, Shah KV (1995) Prevalence of human papillomavirus in cervical cancer: a worldwide perspective. International biological study on cervical cancer (IBSCC) Study Group. J Natl Cancer Inst 87: 796-802

Clifford GM, Smith JS, Plummer M, Munoz N, Franceschi S (2003) Human papillomavirus types in invasive cervical cancer worldwide: a metaanalysis. Br J Cancer 88: 63-73

de Roda Husman AM, Walboomers JM, Hopman E, Bleker OP, Helmerhorst TM, Rozendaal L, Voorhorst FJ, Meijer CJ (1995) HPV prevalence in cytomorphologically normal cervical scrapes of pregnant women as determined by PCR: the age-related pattern. J Med Virol 46: $97-102$

Elfgren K, Rylander E, Radberg T, Strander B, Strand A, Paajanen K, Sjoberg I, Ryd W, Silins I, Dillner J (2005) Colposcopic and histopathologic evaluation of women participating in population-based screening for human papillomavirus deoxyribonucleic acid persistence. Am J Obstet Gynecol 193: 650-657

Forslund O, Antonsson A, Edlund K, van den Brule AJ, Hansson BG, Meijer CJ, Ryd W, Rylander E, Strand A, Wadell G, Dillner J, Johansson B (2002) Population-based type-specific prevalence of high-risk human papillomavirus infection in middle-aged Swedish women. J Med Virol 66: 535-541

Jacobs MV, Snijders PJ, van den Brule AJ, Helmerhorst TJ, Meijer CJ, Walboomers JM (1997) A general primer GP5+/GP6(+)-mediated PCRenzyme immunoassay method for rapid detection of 14 high-risk and 6 low-risk human papillomavirus genotypes in cervical scrapings. J Clin Microbiol 35: 791-795

Khan MJ, Castle PE, Lorincz AT, Wacholder S, Sherman M, Scott DR, Rush BB, Glass AG, Schiffman M (2005) The elevated 10-year risk of cervical precancer and cancer in women with human papillomavirus (HPV) type 16 or 18 and the possible utility of type-specific HPV testing in clinical practice. J Natl Cancer Inst 97: $1072-1079$ women are referred to colposcopy suggesting that verification bias has not materially affected our estimates (Belinson et al, 2001).

It has been suggested that CIN $3+$ is a more appropriate proxy for the risk of future cervical cancer than CIN2 + since the regression rate is higher for CIN2 than for CIN 3 (Schiffman and Kjaer, 2003). Although our statistical power to address CIN3 + rates was more limited, we found similar patterns of differences in HPV-type-specific risks.

In conclusion, we found that different so-called 'high-risk' HPV types convey very different risks for future CIN2+, with HPV 16 and its close relatives HPV 31 and 33 consistently conferring the highest risks. Appreciation of the HPV-type-specific risks for CIN2 + can be helpful for interpretation and design of HPV tests as well as for second-generation vaccines against HPV.
Koutsky LA, Holmes KK, Critchlow CW, Stevens CE, Paavonen J, Beckmann AM, DeRouen TA, Galloway DA, Vernon D, Kiviat NB (1992) A cohort study of the risk of cervical intraepithelial neoplasia grade 2 or 3 in relation to papillomavirus infection. $N$ Engl J Med 327: $1272-1278$

Liaw KL, Glass AG, Manos MM, Greer CE, Scott DR, Sherman M, Burk RD, Kurman RJ, Wacholder S, Rush BB, Cadell DM, Lawler P, Tabor D, Schiffman M (1999) Detection of human papillomavirus DNA in cytologically normal women and subsequent cervical squamous intraepithelial lesions. J Natl Cancer Inst 91: 954-960

Miettinen OS (1974) Proportion of disease caused or prevented by a given exposure, trait or intervention. Am J Epidemiol 99: 325-332

Munoz N, Bosch FX, de Sanjose S, Herrero R, Castellsague X, Shah KV, Snijders PJ, Meijer CJ (2003) Epidemiologic classification of human papillomavirus types associated with cervical cancer. $N$ Engl J Med 348: $518-527$

Peto J, Gilham C, Deacon J, Taylor C, Evans C, Binns W, Haywood M, Elanko N, Coleman D, Yule R, Desai M (2004) Cervical HPV infection and neoplasia in a large population-based prospective study: the Manchester cohort. Br J Cancer 91: $942-953$

Rothman KJ, Greenland S (1998) Modern Epidemiology. Lippincott-Raven: Philadelphia

Schiffman M, Kjaer SK (2003) Chapter 2: natural history of anogenital human papillomavirus infection and neoplasia. J Natl Cancer Inst Monogr 31: 14-19

Schiffman M, Herrero R, Desalle R, Hildesheim A, Wacholder S, Rodriguez AC, Bratti MC, Sherman ME, Morales J, Guillen D, Alfaro M, Hutchinson M, Wright TC, Solomon D, Chen Z, Schussler J, Castle PE, Burk RD (2005) The carcinogenicity of human papillomavirus types reflects viral evolution. Virology 337: 76-84

Sherman ME, Lorincz AT, Scott DR, Wacholder S, Castle PE, Glass AG, Mielzynska-Lohnas I, Rush BB, Schiffman M (2003) Baseline cytology, human papillomavirus testing, and risk for cervical neoplasia: a 10-year cohort analysis. J Natl Cancer Inst 95: 46-52

StataCorp (2005) Stata Statistical Software: Release 9. StataCorp LP: College Station, TX

Szoke K, Sapy T, Krasznai Z, Hernadi Z, Szladek G, Veress G, Dillner J, Gergely L, Konya J (2003) Moderate variation of the oncogenic potential among high-risk human papillomavirus types in gynecologic patients with cervical abnormalities. J Med Virol 71: 585-592

Walboomers JM, Jacobs MV, Manos MM, Bosch FX, Kummer JA, Shah KV, Snijders PJ, Peto J, Meijer CJ, Munoz N (1999) Human papillomavirus is a necessary cause of invasive cervical cancer worldwide. J Pathol 189: $12-19$

Winer RL, Kiviat NB, Hughes JP, Adam DE, Lee SK, Kuypers JM, Koutsky LA (2005) Development and duration of human papillomavirus lesions, after initial infection. J Infect Dis 191: $731-738$ 